\title{
Do antidepressants cause dependence?
}

\author{
PETER M. HADDAD
}

\section{INTRODUCTION}

Most medical authorities do not regard antidepressants as causing dependence, or addiction, but this view has been challenged on the basis that these drugs can cause withdrawal symptoms and that in some patients these symptoms prevent antidepressants being stopped (Medawar, 1997; BBC, October 2002; May 2003, September 2004). This is an important issue for several reasons. First, antidepressants are widely used and so if they cause dependence then large numbers of people could potentially be affected. Second, patients must be given accurate information about drug side effects and so health professionals need to be clear about the issue of dependence. Finally, lay fears about addiction may act as a barrier discouraging those suffering from depression from seeking help or taking prescribed antidepressants. The first part of this paper reviews antidepressant discontinuation, or withdrawal symptoms, with particular emphasis on the clinical features and incidence of such symptoms. The second part of the paper addresses the issue of whether this phenomenon indicates dependence or addiction (the two terms are used synonymously).

With regard to terminology, some authors refer to antidepressant 'withdrawal' syndromes and others to 'discontinuation' syndromes. Both terms refer to the same phenomenon and are likely to continue to be used interchangeably; the main issue is to be clear about the meaning. The term 'withdrawal syndrome' is synonymous, in many people's minds, with addiction or dependence, whereas 'discontinuation syndrome' is less likely to

\footnotetext{
Address for correspondence: Dr. P.M. Haddad, Cromwell House Community Mental Health Centre, Bolton Salford and Trafford Mental Health NHS Trust, Cromwell Road, Eccles, Salford, Manchester M30 OGT (United Kingdom).
}

Declaration of Interests: the author has received honoraria for lecturing and attending advisory boards from the manufacturers of several antidepressants. imply these outcomes. Discontinuation tends to be the preferred term among those who do not regard antidepressants as addictive. However those who view these drugs are additive may regard this term as misleading.

Antidepressant discontinuation symptoms were reported soon after imipramine, the first tricyclic antidepressant, entered clinical practice (Mann \& MacPherson, 1959; Andersen \& Kristiansen, 1959). They occur with antidepressants from all classes including tricyclic antidepressants (TCAs), monoamine reuptake inhibitors (MAOIs), selective serotonin reuptake inhibitors (SSRIs), serotonin and noradrenalin reuptake inhibitors (SNRIs) and miscellaneous antidepressants such as mirtazapine, a noradrenergic and specific serotonergic antidepressant (NaSSA). A recent review identified reports of discontinuation symptoms with 22 antidepressants (Haddad et al., 2004).

\section{CLINICAL FEATURES}

Antidepressant discontinuation reactions have several key features. Symptoms usually appear within a few days of stopping an antidepressant or less commonly reducing the dose. Onset more than 1 week later is unusual. In a naturalistic study of 97 patients the mean interval between stopping an SSRI and the onset of discontinuation symptoms was 2 days (Bogetto et al., 2002). Discontinuation reactions are more common with higher doses and longer courses of treatment and are rare unless treatment has continued for more than 5 weeks. These features suggest that discontinuation symptoms reflect restabilisation of the central nervous system, and other systems, from the adapted state that developed during antidepressant use.

Left untreated, most antidepressant discontinuation reactions are short-lived. In the prospective study by Bogetto et al. (2002) the mean duration of SSRI discontinuation symptoms was 5 days. In a series of 71 untreat- 
ed paroxetine discontinuation reactions reported by doctors as adverse drug reactions (Price et al., 1996), and presumably representing the severer end of the spectrum of reactions, the median duration was 8 days (range 1-52 days). Occasionally symptoms can last several weeks or even months. If the original antidepressant is re-commenced symptoms usually resolve within 24 hours.

Discontinuation symptoms are very variable with over 50 different symptoms being reported with SSRIs (Haddad, 1998). Discontinuation reactions occur on a spectrum in terms of the number and severity of symptoms; reactions range from an isolated symptom to a cluster and from mild to severely disabling. This raises the issue of a 'threshold' for defining a discontinuation syndrome. Although provisional operational criteria for an SSRI discontinuation syndrome have been proposed (Haddad, 1998; Black et al., 2000) there is no accepted definition. The situation is made more complex as different symptom clusters, or discontinuation syndromes, can occur.

A 'general' SSRI discontinuation syndrome has been described (Haddad, 1998; Haddad et al., 2004; Black et al., 2000); the term 'general' is used to differentiate this syndrome from rare SSRI discontinuation syndromes such as mania and extrapyramidal syndromes. Six main symptom groups are recognised within the general SSRI discontinuation syndrome i.e. symptoms of dysequilibrium, sensory abnormalities, gastrointestinal symptoms, general somatic symptoms, sleep disturbance and affective symptoms. The commonest symptoms are dizziness, nausea, lethargy and headache (Haddad, 1998). A similar discontinuation syndrome occurs with venlafaxine (Fava et al., 1997).

No prospective studies have compared discontinuation symptoms in patients stopping antidepressants from different classes making it impossible to comment with certainty on how discontinuation syndromes differs between antidepressant classes. However clinical experience and anecdotal reports suggest that symptoms of dysequilibrium and sensory abnormalities are more prominent with stoppage of SSRIs than with TCAs. In contrast TCA discontinuation reactions seem more likely to involve prominent gastrointestinal symptoms than do SSRI reactions presumably reflecting a greater degree of cholinergic rebound after TCA stoppage.

Several rare discontinuation syndromes have been reported anecdotally (Haddad et al., 2004). These include hypomania and mania (reported with SSRIs, TCAs and MAOIs), extrapyramidal symptoms (reported with TCAs and SSRIs) and cardiac arrhythmias (reported with TCAs).

\section{INCIDENCE}

Several methodological issues are pertinent to the question of incidence of discontinuation syndromes. First high quality research is sparse. Secondly, as already discussed, discontinuation reactions occur on a spectrum in terms of the number and severity of symptoms. Consequently incidence will depend on how symptoms are detected (self report or check list), how symptoms aie defined in terms of severity and duration and the threshold set for defining a syndrome. Thirdly, incidence varies between different antidepressants. Finally a range of confounders will influence incidence e.g. the dose of the antidepressant, the duration of antidepressant treatment, whether the antidepressant is stopped abruptly or tapered and the rate of any taper.

Methodological issues aside, it is apparent that discontinuation symptoms are common with many antidepressants Estimates for the percentage of patients who experience one or more discontinuation symptoms include imipramine $100.0 \%$ (Law et al., 1981), amitriptyline 80.0\% (Bialos et al., 1982), clomipramine 33.3\% (Diamond et al., 1989) and phenelzine 32.2\% (Tyrer, 1984). Turning to newer antidepressants, Fava et al. (1997) reported that during the three days following stoppage of venlafaxine and placebo under double blind conditions, seven $(78 \%)$ of nine venlafaxine treated subjects and two (22\%) of nine placebo-treated patients reported the emergence of adverse events, a statistically significant difference. Among the SSRIs prospective studies indicate that paroxetine is associated with the highest incidence of discontinuation symptoms and fluoxetine the lowest (Rosenbaum et al., 1998; Michelson et al., 2000; Judge et al., 2002; Bogetto et al., 2002; Tint $e t$ al., 2002). Several factors may account for this difference but the long half-life. of fluoxetine and the existence of an active metabolite, norfluoxetine, with an even longer half-life seem important. Abrupt discontinuation of paroxetine leads to approximately $1 / 3$ of patients complaining of one or more discontinuation symptoms on open questioning (Oehrberg et al., 1995) but if symptoms are screened for with a checklist approximately $2 / 3$ of patients experience a 'discontinuation syndrome', defined as 4 or more new or worsened symptoms (Rosenbaum et al., 1998).

In most patients discontinuation symptoms are mild and short lived. However symptoms can cause significant morbidity, they may be misdiagnosed leading to inappropriate treatment, they may adversely effect future antidepressant compliance and they may prevent some patients stopping antidepressant treatment. In the context of this paper, a key question is whether this last effect indicates that antidepressants cause addiction/dependence. 


\section{DO DISCONTINUATION SYMPTOMS INDICATE DEPENDENCE?}

Dependence and addiction are ambiguous words that are used to refer to a range of phenomena by patients and health professionals (Haddad \& Anderson, 1999). When patients ask whether antidepressants are addictive they may refer to one of several concerns; Will they experience withdrawal symptoms?, Will they become tolerant and require escalating doses to keep well?, Will they become a 'junkie' or 'addict' and loose control over their antidepressant use?, Will the antidepressant make them more susceptible to develop depression in the future? In turn, health professionals have distinguished between 'physical' and 'psychological' dependence and neither term necessarily means that a patient will meet the criteria for 'substance dependence' in ICD-10 or DSM-IV, criteria that have evolved from those given in earlier editions. This section examines whether antidepressants cause dependence as defined in ICD-10 and DSM-IV. However the issue of ambiguity is important when communicating with patients and will be returned to in the final section of this paper.

Both DSM-IV (American Psychiatric Association, 1994) and ICD-10 (World Health Organisation, 1992) differentiate between a diagnosis of substance dependence and a drug withdrawal state where substance dependence criteria are not met. In both classification systems substance dependence is a syndrome, withdrawal symptoms are neither sufficient nor mandatory for the diagnosis and behavioural features and a compulsive pattern of drug use underlie most of the diagnostic criteria. In DSM-IV these include excessive time being spent using the drug, inability to control drug use, drug use taking priority over other activities and drug use continuing despite persistent harmful consequences. These features rarely occur in patients taking antidepressants. In terms of DSM-IV and ICD-10 definitions antidepressants in general have no clinically significant potential to cause dependence (Haddad, 1999; Haddad \& Anderson, 1999; Tyrer, 1999). To illustrate this further one can consider some of the DSM-IV dependence criteria in more detail.

One DSM-IV criterion for dependence is excessive time being spent using the drug, obtaining it or recovering from its effects. It is virtually unheard of for patients to try and obtain additional antidepressant prescriptions by forgery, registering simultaneously with more than one GP or presenting to hospital A\&E departments with fabricated stories of needing an emergency supply of an antidepressant; in contrast all these scenarios are familiar to doctors in the case of opiates and benzodiazepines, two drugs where iatrogenic addiction or dependence is recognised. Another DSM-IV dependence criterion is that use of the drug exceeds that which was intended. This is well illustrated by some alcohol dependent patients who report that once they start drinking they cannot stop. Such loss of control is not recognised with antidepressants. A third DSM-IV criterion is tolerance. Randomised controlled trials indicate that many patients continue to derive prophylactic benefit from a constant antidepressant dose over several years (Frank et al., 1990). Although some patients do need to increase their antidepressant dose due to a relapse of illness, it is simplistic to automatically assume that this indicates tolerance; the effect could also be explained in terms of increasing psychosocial or organic stressors that underlie the illness.

Antidepressants apart, many drugs cause discontinuation symptoms but are not associated with addiction or a dependence syndrome e.g. anticonvulsants, beta-blockers, nitrates, diuretics, centrally acting antihypertensives, sympathomimetics, heparin (Routledge \& Bialas, 1997), tamoxifen (Kerr \& Myers, 1999), dopaminergic agents (Keyser \& Rodnitzky, 1991), and antipsychotics (Gardos et al., 1978; Tranter \& Healy, 1998). Conversely some highly addictive drugs, including freebase (crack) cocaine, cause only relatively minor withdrawal syndromes.

Other evidence supports the view that antidepressants are not addictive. First, antidepressants are widely used in patients with drug and alcohol dependence to treat comorbid depression and in some cases to attempt to treat the substance dependence. One would expect this group of patients to be particularly susceptible to become addicted to antidepressants if there was such a potential but such problems are not reported. Second, in animal models antidepressants are not self-administered whereas addictive drugs such as benzodiazepines and opiates are (Schindler et al., 2002; Gomez et al., 2002). Third, most addictive drugs have some immediate pleasurable effect after initial consumption (e.g. relaxation with alcohol, excess energy with amphetamines, euphoria with opiates) but this is not the case with antidepressants. Finally, most addictive prescription drugs have a 'black market' or 'street' value and will be 'diverted' or sold on for use for non-medical reasons (Nutt, 2003). Neither phenomenon is seen to any significant extent with antidepressants.

Two exceptions to the rule that antidepressants are not addictive are amineptine and tranylcypromine, rarely prescribed antidepressants with dopaminergic effects (Haddad, 1999). Case reports identified the addictive potential of both drugs shortly after they entered clinical practice, something that has not happened with other antidepressants despite these being prescribed in far greater amounts. Stimulant effects are likely to account for the dependence noted with amineptine and tranylcypromine, and, as most antidepressants have no such effects they appear to be the exceptions that prove the rule. 
In conclusion antidepressants, with the exception of amineptine and tranylcypromine, have no significant potential to cause dependence as defined in DSM-IV or ICD-10 though discontinuation symptoms are common.

\section{INTRACTABLE DISCONTINUATION SYMPTOMS}

Most patients who experience antidepressant discontinuation symptoms have mild and short-lived symptoms. Of those that develop problematic discontinuation symptoms, most can be withdrawn from their antidepressant with support and a gradual taper or, in the case of SSRIs, by switching to fluoxetine (Haddad et al., 2004). However several patient websites contain multiple self-reports of patients in whom discontinuation symptoms prevented antidepressant stoppage or made coming off medication difficult and distressing. Similar effects occasionally occur with antipsychotics. Unfortunately there is no epidemiological research to indicate the size of this problem or why some patients experience severe problems when most do not. As these patients do not fulfil current criteria for dependence, they cannot be regarded as being dependent on, or addicted to, their antidepressant. Some may regard this differentiation as semantic and argue that if a patient cannot stop a drug when they want to they are dependent on it irrespective of current definitions. In answer to this argument two related points can be made.

First, there is a need to be precise about diagnoses in medicine and particularly in psychiatry where there are few laboratory diagnostic tests and diagnosis is largely based on clinical syndromes. If diagnostic boundaries become blurred, then diagnoses become meaningless in that they cannot convey useful information to patients and health professionals about the condition. Furthermore, 'blurred' diagnoses will not define homogenous groups that can aid research.

Second, stating that antidepressants are not associated with dependence, as defined in ICD-10 and DSM-IV, has practical implications that remain irrespective of whether discontinuation symptoms make antidepressant stoppage difficult. For example, antidepressants are not associated with tolerance or patients loosing control over their use of medication. Furthermore once discontinuation symptoms have resolved, a patient will not crave their antidepressant or feel compelled to restart it. This is of great importance, dependence as described in ICD-10 and DSM-IV is a relapsing condition; when withdrawal symptoms have ceased patients still crave their drug and often relapse into further drug taking, sometimes after long periods of abstinence. It is the relapsing nature of dependence that makes it so problematic for patients and pro- fessionals; managing the withdrawal symptoms of addictive drugs, such as alcohol or opiates, is not in itself a major problem.

\section{WAYS FORWARD}

There is a danger that the debate over antidepressants and dependence becomes a semantic argument, one side arguing that these drugs do not cause dependence in terms of current definitions and the other side replying that these definitions are not designed to detect the type of dependence or addiction that occurs with antidepressants. The fact that 'addiction' and 'dependence' are ambiguous words, that mean different things to different people and cover a range of phenomena, adds to the confusion. One way round this is to ensure that doctors are more explicit in the information that they give to patients and do not simply rely on using the 'umbrella' terms of 'addiction' and 'dependence' alone. Such information should be provided routinely to all patients who are considering starting an antidepressant and should include the following:

1. Antidepressants are not associated with tolerance i.e. for most people the dose that gets you well, keeps you well and there is no need to continually escalate the dose to maintain the benefit.

2. Patients do not crave antidepressants either when taking them or after courses have finished.

3. Patients do not lose control over their antidepressant use so that taking these drugs becomes chaotic or dominates their lives.

4. For the above reasons antidepressants are not addictive in the way that alcohol and illicit drugs, such as amphetamines and heroin, are.

5. Antidepressants do not make you more likely to suffer from depression in the future.

6. Many patients who suddenly stop or interrupt their antidepressant will experience discontinuation or withdrawal symptoms. These usually start one or two days later, and with SSRIs the commonest symptoms are dizziness, headache, nausea and lethargy. These symptoms usually resolve within a week without any treatment but if the antidepressant is restarted they revolve within hours. Occasionally discontinuation symptoms can be severe, persist for weeks and in some cases they have prevented patients stopping their antidepressant. It is unclear how often this occurs but it seems to be the exception not the norm.

7. To prevent discontinuation/ withdrawal symptoms antidepressant should be taken regularly; missing doses out can cause discontinuation symptoms. When antidepressants are to be stopped the dose should be 
reduced gradually over several weeks to minimise or prevent discontinuation symptoms occurring.

Patients need to be given additional information about antidepressants including the likelihood of benefit, the delayed onset of action and the gradual nature of improvement thereafter. They also require information about their underlying psychiatric illness and alternative treatments to antidepressants so that they can make an informed decision about treatment. In summary information about antidepressants and dependence is only one part of a package of information that patients considering antidepressant treatment require.

Patients who experience severe discontinuation symptoms or find that they cannot stop their antidepressant due to such symptoms require appropriate support and management. Research is required to investigate the severity and duration of antidepressant discontinuation reactions in a naturalistic cohort of patients and determine the proportion who are unable to stop treatment. If such patients could be predicted they could be offered alternative treatments.

\section{REFERENCES}

American Psychiatric Association (1994). Diagnostic and Statistical Manual of Mental Disorder, 4th ed. American Psychiatric Association: Washington DC.

Andersen H. \& Kristiansen E.S. (1959). Tofranil treatment of endogenous depressions. Acta Psychiatrica Scandinavica 34, 387-397.

Bialos D., Giller E., Jatlow P., Docherty J. \& Harkness L. (1982). Recurrence of depression after discontinuation of amitriptyline. American Journal of Psychiatry139, 325-329.

Black K., Shea C., Dursun S. \& Kutcher S. (2000). Selective serotonin reuptake inhibitor discontinuation syndrome: proposed diagnostic criteria. Journal of Psychiatry Neuroscience 25, 255-261.

Bogetto F., Bellino S., Revello R.B. \& Patria L. (2002). Discontinuation syndrome in dysthymic patients treated with selective serotonin reuptake inhibitors: a clinical investigation. CNS Drugs 16, 273283.

Diamond B.I., Borison R.L., Katz R. \& DeVeaugh-Geiss J. (1989). Rebound reactions due to clomipramine. Psychopharmacology Bulletin 255, 209-212.

Fava M., Mulroy R., Alpert J., Nierenberg A.A. \& Rosenbaum J.F. (1997). Emergence of adverse effects following discontinuation of treatment with extended-release venlafaxine. American Joumal of Psychiatry 154, 1760-1762.

Frank E., Kupfer D.J., Perel J.M., C. Cornes, D.B. Jarrett, A.G. Mallinger, M.E. Thase, A.B. McEachran \& V.J. Grochocinski (1990). Three-year outcomes for maintenance therapies in recurrent depression. Archives of General Psychiatry 47 1093-1099.

Gardos G., Cole J.O. \& Tarsy D. (1978). Withdrawal syndromes associated with antipsychotic drugs. American Journal of Psychiatry $135,1321-1324$.

Gomez T.H., Roache J.D. \& Meisch R.A. (2002). Orally delivered alprazolam, diazepam and triazolam as reinforcers in rhesus monkeys. Psychopharmacology (Berl) 161, 89-94.

Haddad P. (1998). The SSRI discontinuation syndrome. Journal of Psychopharmacology 12, 305-313.

Haddad P. (1999). Do antidepressants have any potential to cause addi- tion? Journal of Pychopharmacology 13, 300-307.

Haddad P. \& Anderson I. (1999). Antidepressants aren 't addictive: clinicians have depended on them for years. Journal of Psychopharmacology 13, 291-292.

Haddad P.M., Anderson I. \& Rosenbaum J. (2004). Antidepressant discontinuation syndromes. In Adverse Syndromes and Psychiatric Drugs; a Clinical Guide (ed. P. Haddad, S. Dursun and B. Deakin, pp. 183-205. Oxford University Press: Oxford.

Judge R., Parry M.G., Quail D. \& Jacobson J.G. (2002). Discontinuation symptoms: comparison of brief interruption in fluoxetine and paroxetine treatment. International Clinical Psichopharmacology 17, 217-225.

Law W., Petti T.A. \& Kazdin A. (1981). Withdrawal symptoms after gradual cessation of imipramine in children. American Joumal of Psychiatry 118, 647-650

Kerr B. \& Myers P. (1999). Withdrawal syndrome following long-term administration of tamoxifen. Journal of Psychopharmacology 13, 419.

Keyser D.L. \& Rodnitzky R.L. (1991). Neuroleptic malignant syndrome in Parkinon's disease after withdrawal or alteration of dopaminergic therapy. Archives of Internal Medicine 151, 794-796.

Mann A.M. \& MacPherson A (1959). Clinical experience with imipramine (G22355) in the treatment of depression. Canadian Psychiatric Association Journal 4, 38-47.

Medawar C. (1997). The Antidepressant Web - marketing depression and making medicines work. Intemational Joumal of Risk and Safety Medicine 10, 75-126.

Michelson D., Fava M., Amsterdam J., Apter J., Londborg P., Tamura R. \& Tepner R.G., (2000). Interruption of selective serotonin reuptake inhibitor treatment. Double-blind, placebo-controlled trial. British Journal of Psychiatry 176, 363-368.

Nutt D. (2003). Death and dependence: current controversies over the selective serotonin reuptake inhibitors. Journal of Psychopharmacology 17, 355-364.

Oehrberg S., Christiansen P.E., Behnke K., Borup A.L., Severin B., Soegaard J., Calberg H., Judge R., Ohrstrom J.K. \& Manniche P.M. (1995). Paroxetine in the treatment of panic disorder. A randomized, double-blind, placebo-controlled study. British Journal of Psychiatry 167, 374-379.

Price J.S., Waller P.C., Wood S.M \& MacKay A.V. (1996). A comparison of the post-marketing safety of four selective serotonin reuptake inhibitors, including the investigation of symptoms occurring on withdrawal. British Journal of Clinical Pharmacology 42, 757763.

Rosenbaum J.F., Fava M., Hoog S.L., Ascroft R.C. \& Krebs W.B. (1998). Selective serotonin reuptake inhibitor discontinuation syndrome: a randomised clinical trial. Biological Psychiatry 44, 77-87.

Routledge P.A. \& Bialas M.C. (1997). Adverse reactions to drug withdrawal. Adverse Drug Reaction Bulletin 187, 711-714.

Schindler C.W., Panlilio L.V. \& Goldberg S.R. (2002). Second-order schedules of drug self-administration in animals. Psychopharmacology (Berl.) 163, 327-344.

Tint A.K., Haddad P.M., Anderson I.M. \& Tiplady B. (2002). SSRI/venlafaxine discontinuation symptoms during aptidepressant switching: an interim analysis. Journal of Psychopharmacology, Suppl. 16(3), A44.

Tranter R. \& Healy D. (1998). Neuroleptic discontinuation syndromes. Journal of Psychopharmacology 12, 401-406.

Tyrer P. (1984). Clinical effects of abrupt withdrawal from tricyclic antidepressants and monoamine oxidase inhibitors after long term treatment. Journal of Affective Disorders 6, 1-7.

Tyrer P. (1999). Stress diathesis and pharmacological dependence. Jourmal of Psychopharmacology 13, 294-295.

World Health Organization (1992). The ICD-10 Classification of Mental and Behavioural Disorders. World Health Organization: Geneva. 